\title{
Complement Activation in Patients With Diabetic Nephropathy
}

\author{
Pascal Bus ${ }^{1,5}$, Jamie S. Chua ${ }^{1,5}$, Céline Q.F. Klessens ${ }^{1}$, Malu Zandbergen ${ }^{1}$, Ron Wolterbeek ${ }^{2}$, \\ Cees van Kooten ${ }^{3}$, Leendert A. Trouw ${ }^{4}$, Jan A. Bruijn ${ }^{1}$ and Hans J. Baelde ${ }^{1}$ \\ ${ }^{1}$ Department of Pathology, Leiden University Medical Center, Leiden, The Netherlands; ${ }^{2}$ Department of Medical Statistics and \\ Bioinformatics, Leiden University Medical Center, Leiden, The Netherlands; ${ }^{3}$ Department of Nephrology, Leiden University \\ Medical Center, Leiden, The Netherlands; and ${ }^{4}$ Department of Immunohematology and Blood Transfusion, Leiden University \\ Medical Center, Leiden, The Netherlands
}

Introduction: Complement activation plays a role in various organs in patients with diabetes. However, in diabetic nephropathy (DN), the role of complement activation is poorly understood. We examined the prevalence and clinical significance of complement deposits in the renal tissue of cases with type 1 and type 2 diabetes with and without DN.

Methods: We measured the prevalence of glomerular C4d, C1q, mannose-binding lectin (MBL), and C5b-9 deposits in 101 autopsied diabetic cases with DN, 59 autopsied diabetic cases without DN, and 41 autopsied cases without diabetes or kidney disease. The presence of complement deposits was scored by researchers who were blinded with respect to the clinical and histological data.

Results: C4d deposits were more prevalent in cases with DN than in cases without DN in both the glomeruli ( $46 \%$ vs. $26 \%)$ and the arterioles $(28 \%$ vs. $12 \%)$. C1q deposits were also increased in the glomerular hili $(77 \%$ vs. $55 \%)$ and arterioles (33\% vs. $14 \%)$, and were correlated with DN $(P<0.01)$. MBL deposits were only rarely observed. C5b-9 deposits were more prevalent in the cases with diabetes mellitus (DM) than in the cases without DM (69\% vs. 32\%; $P<0.001)$. Finally, glomerular C4d and C5b-9 deposits were correlated with the severity of $\operatorname{DN}(\rho=0.341$ and 0.259 , respectively; $P<0.001)$.

Conclusion: Complement activation is correlated with both the presence and severity of DN, suggesting that the complement system is involved in the development of renal pathology in patients with diabetes and is a promising target for inhibiting and/or preventing DN in these patients.

Kidney Int Rep (2018) 3, 302-313; https://doi.org/10.1016/j.ekir.2017.10.005

KEYWORDS: C4d; complement activation; diabetic nephropathy; histological lesions; renal pathology

(C) 2017 International Society of Nephrology. Published by Elsevier Inc. This is an open access article under the CC BYNC-ND license (http://creativecommons.org/licenses/by-nc-nd/4.0/).

iabetic nephropathy (DN), the leading cause of end-stage renal disease worldwide, can occur in patients with either type 1 or type 2 diabetes mellitus (DM). ${ }^{1-3} \mathrm{DN}$ is characterized by a gradual increase in proteinuria and blood pressure, and a gradual decrease in glomerular filtration rate that may result in the need for renal replacement therapy. Prolonged hyperglycemia can lead to the development of $\mathrm{DN}$ via a number of pathways with complex interactions; however, the precise cellular and molecular mechanisms that underlie this process are poorly understood. ${ }^{4-6}$

The first evidence for a possible role of the complement system in the development of DN was provided by the

Correspondence: Pascal Bus, Department of Pathology, Leiden University Medical Center, L1Q, room P0-107, P.O. Box 9600, 2300 RC Leiden, The Netherlands. E-mail: p.bus@lumc.nl

${ }^{5} \mathrm{~PB}$ and JSC contributed equally to this work.

Received 19 September 2017; accepted 9 October 2017; published online 14 October 2017 finding that serum, urine, and renal samples obtained from patients with diabetes often contained activated complement proteins, and that these proteins are associated with DN. ${ }^{7-14}$ Serum levels of mannose-binding lectin (MBL) are correlated with the severity of DN, which suggest a role for the lectin complement pathway. ${ }^{15} \mathrm{In}$ addition, advanced glycation end-products can directly bind $\mathrm{Clq}$ and activate the complement system, ${ }^{16}$ and DN has been associated with increased renal expression of complement factors $\mathrm{C} 3, \mathrm{C} 4$, and $\mathrm{C} 9$ (at the protein level), as well as increased expression of $\mathrm{Clq}, \mathrm{Cls}$, and $\mathrm{Clr}$ (at the mRNA level), which also suggest a role for the classical complement pathway. ${ }^{8,17}$ Furthermore, hyperglycemia can cause glycation-induced dysfunction and/or inactivation of complement regulatory proteins, including CD59, which inhibits C5b-9 under physiological conditions $^{11,18}$; this glycation-induced complement dysregulation leads to increased C5b-9 levels in patients with $\mathrm{DN}{ }^{11}$ In a rat model of type 2 diabetes, treating diabetic rats with a $\mathrm{C} 3 \mathrm{a}$ receptor antagonist improved 
renal function and reduced both albuminuria and the deposition of extracellular matrix proteins. ${ }^{19}$

Recently, 2 groups reviewed the role of complement activation in DM. ${ }^{7,14}$ They concluded that the relative role of complement in the development of DM-related complications, including DN, are unknown. Moreover, it remains unclear whether these mechanisms are similar between type $1 \mathrm{DM}$ and type $2 \mathrm{DM}^{7}{ }^{7}$ To address these questions, we examined whether complement activation occurred in renal autopsy samples obtained from a large cohort of cases with diabetes with and without DN. Specifically, we measured the prevalence, localization, and staining patterns of renal $\mathrm{C} 4 \mathrm{~d}$ and C5b-9 deposits. Furthermore, because both the lectin and classical complement pathways could lead to $\mathrm{C} 4 \mathrm{~d}$ deposits, and because both pathways might be involved in the development of DN, we also determined which complement pathway was associated with the deposition of C4d in cases with DN. We validated our findings in renal biopsy samples. Finally, we correlated complement deposition with histopathology, and examined differences in complement deposition between cases with type 1 and 2 DM.

\section{METHODS}

This study group includes a selection of a previously described cohort. $^{20}$ In brief, we retrospectively searched the database of our pathology department for native kidneys from adult cases with either type 1 or type 2 DM who were autopsied from 1984 to 2004. We initially included 184 autopsied kidneys that were prepared for light microscopy, electron microscopy, and immunohistochemistry. We subsequently excluded 25 cases due to poor tissue quality or missing tissue for immunostaining. Thus, we included a total of 159 samples from cases with diabetes for whom we confirmed the histopathological presence or absence of $\mathrm{DN}$ according to the classification for $\mathrm{DN} .{ }^{21}$ In addition, we included a control group consisting of autopsy samples obtained from 41 cases without diabetes without renal pathology. We validated our findings with autopsy tissue by examining 12 kidney biopsies from patients with DN and 10 biopsies obtained from healthy living transplantation donors.

\section{Clinical Data}

The clinical information was obtained retrospectively via the medical records and autopsy reports available at Leiden University Medical Center, and the general practitioners of the patients. The following laboratory parameters were collected from a period starting 1 year before the patient died: serum creatinine, estimated glomerular filtration rate (eGFR) (calculated using the Modification of Diet in Renal Disease formula), microalbuminuria (defined as $30-300 \mathrm{mg} / \mathrm{l}$ ), proteinuria (defined as $>300 \mathrm{mg} / \mathrm{l}$ ) measured via a 24 hour urine or dipstick test, systolic and diastolic blood pressures, serum hemoglobin, serum cholesterol, and serum glycosylated hemoglobin $\left(\mathrm{HbA}_{\mathrm{lc}}\right) \cdot{ }^{20}$ The clinical data were analyzed to reflect a stable representation of the serum and/or urine levels, thereby excluding data that were clearly affected by an unstable clinical condition (for example, patients who were clinically unstable in an intensive care unit before death). Cause of death was categorized into the following 5 general categories: cancer, cardiovascular, infection and/or sepsis, multiple pathologies, and other (e.g., high-impact trauma).

\section{Histopathology and Transmission Electron Microscopy}

Renal tissue was fixed in $10 \%$ buffered formalin and embedded in paraffin. Sections were cut and then stained with hematoxylin and eosin, periodic-acid Schiff, and silver using standard protocols. Glomerular lesions, interstitial lesions, and vascular lesions were scored according to the histopathological classification for $\mathrm{DN}^{21}$ Discrimination between class 0 (i.e., no DN) and class I DN was determined using transmission electron microscopy, as described previously. ${ }^{20}$

\section{Immunohistochemistry and Immunofluorescence}

To measure renal complement activation, immunohistochemistry was performed on adjacent kidney sections using primary antibodies against the following proteins: C4d (1:150; Biomedica Gruppe, Vienna, Austria), which is a cleavage product of C4 that binds covalently to the target tissue and can arise from both the classical and MBL pathways; Clq (1:1200; DakoCytomation, Glostrup, Denmark), which reflects activation of the classical complement pathway; MBL (1:300; SigmaAldrich Biotechnology, St. Louis, Missouri), which reflects activation of the MBL pathway; and SC5b-9 (1:500; Quidel, San Diego, California), which is formed by activation of any of the aforementioned pathways. To investigate the presence of natural antibodies, immunofluorescence was performed on sections using a fluorescein isothiocyanate-labeled anti-IgM antibody (1:20; DakoCytomation).

The immunostained tissue samples were scored semiquantitatively as follows: staining of glomeruli was scored as absent (representing either an absence of staining or trace levels of staining in $<5 \%$ of glomeruli) or present (representing staining in $\geq 5 \%$ of glomeruli). If present, the distribution of glomerular staining was scored as focal $(5 \%-50 \%$ of the glomeruli) or diffuse $(>50 \%$ of the glomeruli), and the staining pattern was 
scored as segmental ( $<50 \%$ of the glomerular tuft) or global $(>50 \%$ of the glomerular tuft). In addition, if present, glomerular staining was scored as present in the glomerular capillary walls, mesangial cells, or both. Immunohistochemical staining in the glomerular hilus, arterioles, and arterial branches, was scored as absent or present (i.e., the presence of staining in $\geq 1$ glomerular hilus, arteriole, and/or arterial branch was scored as positive). Renal tissue specimens containing $\geq 100$ glomeruli were scored by 2 investigators who were blinded with respect to the clinical data of the cases.

\section{Statistical Analysis}

The SPSS statistical software package (version 20.0; IBM, Armonk, NY) was used for all statistical analyses. Categorical variables were compared using the $\chi^{2}$ test or Fisher exact test, where appropriate. Continuous variables were compared using the Student's $t$-test or the Mann-Whitney $U$ test, where appropriate. Spearman rank correlation coefficient was used to analyze the correlation between diabetes class and the presence of glomerular complement deposits. Differences with a $P$ value $<0.05$ were considered statistically significant. All tissue samples were coded, and then handled and analyzed anonymously in accordance with the Declaration of Helsinki. Approval for the study was obtained from the medical ethics committee of Leiden University Medical Center.

\section{RESULTS}

\section{Clinical and Histological Characteristics}

We included 159 diabetic cases; histologically confirmed DN was present in 101 cases (64\%) and absent in 58 cases $(36 \%)$. We also included an age- and sex-matched control cohort of 41 renal samples from autopsied nondiabetic cases without renal pathology. The clinical characteristics of the cases are summarized in Table 1. The duration of diabetes was significantly higher in the cases with DN than in the cases without $\mathrm{DN}(P=0.017)$; however, we found no difference between these 2 groups with respect to age, sex, diabetes type, presence of hypertension, serum creatinine, eGFR, or $\mathrm{HbA}_{\mathrm{lc}}$ levels.

The histological features of the cases with diabetes are summarized in Table 2. Among the 101 cases with DN, DN was distributed as follows: $20 \%$ with class I, $20 \%$ with class IIA, $10 \%$ with class IIB, $45 \%$ with class III, and 5\% with class IV. Compared with the cases without DN, the cases with DN had a significantly higher prevalence of glomerular hyalinosis, glomerular capsular drop, arteriosclerosis, and arteriolar hyalinosis $(P<0.05)$. In addition, the cases with DN had more interstitial fibrosis and tubular atrophy (IFTA) compared with the cases without DN $(P=0.028)$.

\section{C4d Deposition Is Associated With DN}

C4d deposits in the glomeruli, glomerular hili, and arterioles were significantly more prevalent in cases with diabetes than in the control cases without diabetes

Table 1. Clinical characteristics of the control cases and diabetic cases

\begin{tabular}{|c|c|c|c|c|c|}
\hline Clinical characteristics & $\begin{array}{l}\text { Nondiabetic controls } \\
\quad(n=41)\end{array}$ & $\begin{array}{l}\text { Diabetic cases without DN } \\
\qquad(n=58)\end{array}$ & $\begin{array}{l}\text { Diabetic cases with DN } \\
\qquad(n=101)\end{array}$ & $\begin{array}{l}\text { All diabetic cases patients } \\
\qquad(n=159)\end{array}$ & $P$ value \\
\hline Age, yr & $63.8 \pm 16.8$ & $69.1 \pm 12.6$ & $69.0 \pm 12.8$ & $69.1 \pm 12,7$ & $0.100^{a}$ \\
\hline Female, n (\%) & $16(39)$ & $24(41)$ & $47(47)$ & $71(45)$ & $0.754^{a}$ \\
\hline Duration of diabetes, $\mathrm{yr}$ & NA & $8.4 \pm 7.3$ & $15.3 \pm 13.1$ & $13.3 \pm 10.0$ & $0.003^{b}$ \\
\hline TIDM (median, IQR) & NA & $17.5(2)$ & $28.0(33)$ & $18.0(28)$ & $0.215^{b}$ \\
\hline T2DM (median, IQR) & NA & $5.0(5)$ & $10.0(14)$ & $8.0(15)$ & $0.004^{b}$ \\
\hline eGFR, $\mathrm{ml} / \mathrm{min}$ per $1.73 \mathrm{~m}^{2}$ & NA & $59 \pm 36$ & $50 \pm 33$ & $54 \pm 34$ & $0.184^{b}$ \\
\hline $\mathrm{HbA}_{1 \mathrm{C}}$ \% unit & NA & $7.5 \pm 1.8$ & $8.5 \pm 2.4$ & $8.1 \pm 2,3$ & $0.117^{\mathrm{b}}$ \\
\hline Hypertension, n (\%) & NA & $27 / 48(56)$ & $44 / 85(52)$ & 71/133 (53) & $0.618^{b}$ \\
\hline Systolic pressure, mm Hg & NA & $134 \pm 29$ & $155 \pm 29$ & $135 \pm 29$ & $0.780^{\mathrm{b}}$ \\
\hline Diastolic pressure, $\mathrm{mm} \mathrm{Hg}$ & NA & $75 \pm 14$ & $75 \pm 12$ & $75 \pm 13$ & $0.961^{b}$ \\
\hline Cause of death, $\mathrm{n}(\%)$ & & & & & $0.199^{a}$ \\
\hline Other & $4(10)$ & $8(14)$ & $18(18)$ & $26(16)$ & \\
\hline
\end{tabular}

DN, diabetic nephropathy; eGFR, estimated glomerular filtration rate; $\mathrm{HbA}_{1 \mathrm{C}}$, glycosylated hemoglobin; IQR, interquartile range; T1DM, type 1 diabetes mellitus; T2DM, type 1 diabetes mellitus.

Data are presented as the mean $\pm S D$, unless stated otherwise

${ }^{a}$ Between nondiabetic controls without renal disease, diabetic cases without DN and diabetic cases with DN.

${ }^{\mathrm{b}}$ Between diabetic cases without DN and diabetic cases with DN. 
Table 2. Histological characteristics of the diabetic cases

\begin{tabular}{|c|c|c|c|c|}
\hline $\begin{array}{l}\text { Histological } \\
\text { characteristics }\end{array}$ & $\begin{array}{c}\text { Diabetic cases } \\
\text { without DN } \\
(n=58)\end{array}$ & $\begin{array}{c}\text { Diabetic cases } \\
\text { with DN } \\
(n=101)\end{array}$ & $\begin{array}{l}\text { All cases } \\
\text { diabetic } \\
\text { patients } \\
(n=159)\end{array}$ & $P$ value ${ }^{a}$ \\
\hline \multicolumn{5}{|l|}{ DN class } \\
\hline 0 (no DN) & $58(100)$ & $0(0)$ & $58(37)$ & NA \\
\hline I & $0(0)$ & $20(20)$ & $20(13)$ & \\
\hline$\| \mathrm{A}$ & $0(0)$ & $21(20)$ & $21(13)$ & \\
\hline IIB & $0(0)$ & $10(10)$ & $10(6)$ & \\
\hline III & $0(0)$ & $45(45)$ & $45(28)$ & \\
\hline IV & $0(0)$ & $5(5)$ & $5(3)$ & \\
\hline Glomerular hyalinosis & $19(33)$ & $85(84)$ & $104(65)$ & $<0.001$ \\
\hline Glomerular capsular drop & $2(3)$ & $15(15)$ & $17(11)$ & 0.025 \\
\hline FSGS & $2(3)$ & $12(12)$ & $14(9)$ & 0.071 \\
\hline \multicolumn{5}{|l|}{ IFTA } \\
\hline Absent & $22(38)$ & $17(17)$ & $39(25)$ & 0.028 \\
\hline $10 \%-25 \%$ & $25(43)$ & $56(55)$ & $81(51)$ & \\
\hline $25 \%-50 \%$ & $5(9)$ & $15(15)$ & $20(13)$ & \\
\hline$>50 \%$ & $6(10)$ & $13(13)$ & $19(12)$ & \\
\hline Arteriosclerosis & $46(79)$ & $92(91)$ & $138(87)$ & 0.035 \\
\hline Arteriolar hyalinosis & $46(79)$ & $92(91)$ & $138(87)$ & 0.035 \\
\hline Cholesterol emboli & $3(5)$ & $3(3)$ & $6(4)$ & 0.483 \\
\hline
\end{tabular}

DN, diabetic nephropathy; FSGS, focal segmental glomerulosclerosis; IFTA, interstitial fibrosis and tubular atrophy.

${ }^{a}$ Between diabetic cases without DN and diabetic cases with DN.

Data are presented as the number of cases (\%).

$(P<0.05)$ (Table 3), in contrast to C4d deposits in arterial branches $(P=0.171)$ (Figure 1$)$. In the diabetic cohort, C4d deposits were present in the glomeruli, glomerular hili, and arterioles in $38 \%, 48 \%$, and $22 \%$ of the cases, respectively. In the nondiabetic control cohort, C4d deposits were rarely observed in any vascular structure.

In the cohort of cases with diabetes, cases with DN had a significantly higher prevalence of C4d deposits in the glomeruli and arterioles than cases without DN (Figure 1) $(P=0.019$ and $P=0.022$, respectively). The cases with DN had a higher prevalence of C4d in the glomerular capillary walls ( $45 \%$ vs. $26 \%$ of cases without DN; $P=$ $0.019)$ and in the mesangial cells $(26 \%$ vs. $12 \%$, respectively; $P=0.041$ ) than cases without DN. The distribution of glomerular C4d did not differ significantly between the 2 groups. However, the global staining pattern of C4d was significantly more prevalent in cases with DN than cases without DN $(15 \%$ vs. $5 \% ; P=0.044)$.

Within our cohort of cases with DN, eGFR was significantly lower in the cases with glomerular C4d than in the cases without glomerular C4d $(39.8 \pm 28.6 \mathrm{ml} / \mathrm{min}$ per $1.73 \mathrm{~m}^{2}$ vs. $60.3 \pm 33.5 \mathrm{ml} / \mathrm{min}$ per $1.73 \mathrm{~m}^{2}$; respectively; $P=0.004)$. In contrast, we found no other correlation between $\mathrm{C} 4 \mathrm{~d}$ deposits and clinical data (Table 4).

\section{Evidence for Classical Complement Activation in Cases With Diabetes}

Next, to investigate which complement pathway(s) led to the deposition of $\mathrm{C} 4 \mathrm{~d}$ in cases with $\mathrm{DN}$, we stained

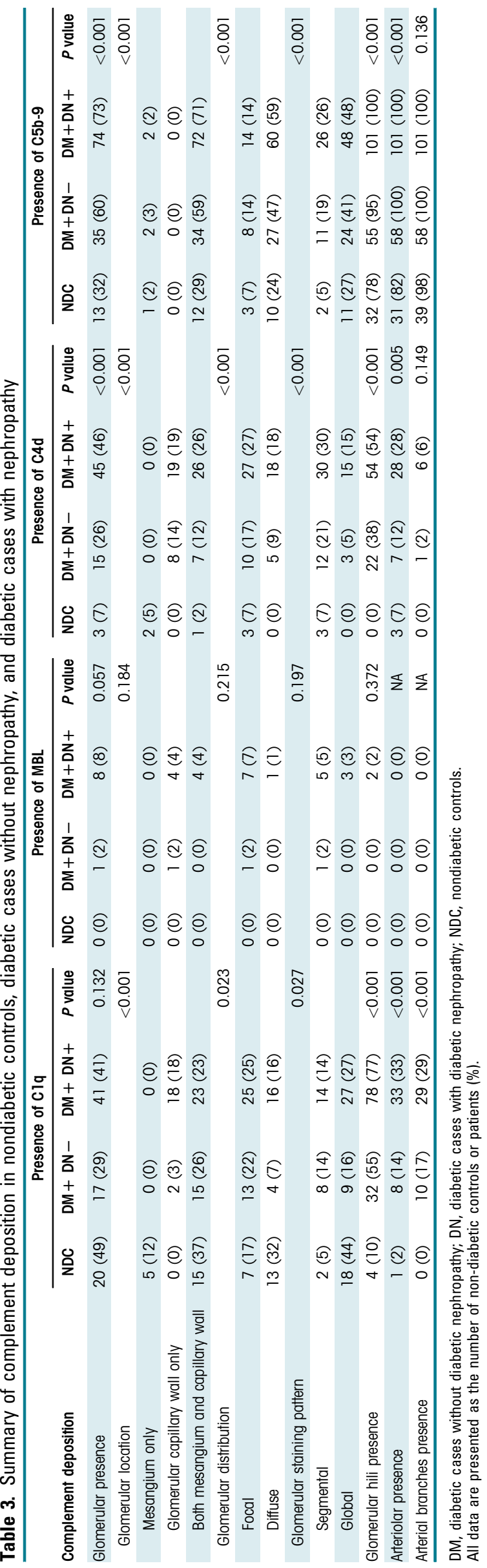




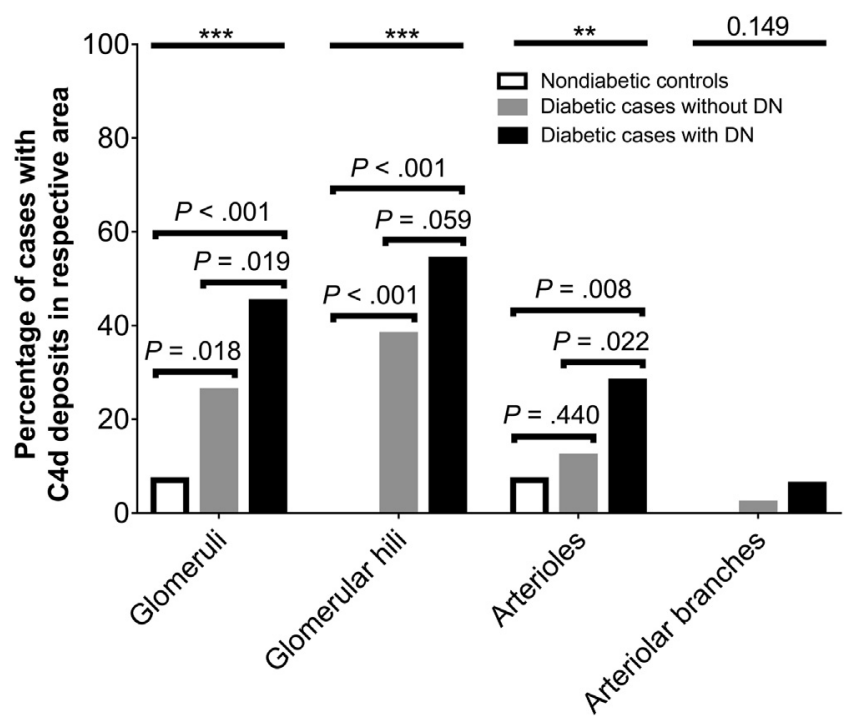

Figure 1. Prevalence of C4d deposits in cases and controls. The percentage of cases and controls with complement factor C4d is shown for the indicated renal structures. Asterisks represent the overall differences among the nondiabetic controls, the diabetic cases without diabetic nephropathy (DN), and the diabetic cases with DN. The $P$ values shown between the 2 groups represent post hoc analyses. ${ }^{* *} P<0.01$ and ${ }^{* *} P<0.001$.

renal tissue for MBL (to measure the lectin pathway) and $\mathrm{Clq}$ (to measure the classical pathway).

MBL was only observed in $6 \%$ of the kidneys from cases with diabetes and was not observed in the control cases without diabetes. When present, the staining pattern of MBL in the glomeruli was predominantly focal and segmental; MBL was not observed in the glomerular hili, arterioles, or arterial branches. We found no significant differences between MBL deposition in the cases with diabetes and control cases without diabetes or between the cases with diabetes with DN and the cases with diabetes without DN (Figure 2a and Table 3).

Glomerular $\mathrm{Clq}$ was present in $36 \%$ of the cases with diabetes, and the staining pattern was predominantly focal and global. The prevalence of glomerular $\mathrm{Clq}$ was not significantly different between the cases with diabetes and control cases without diabetes $(37 \%$ vs. $49 \%$, respectively; $P=0.150$ ) (Figure $2 \mathrm{~b}$ and Table 3). In contrast, the prevalence of $\mathrm{Clq}$ in the glomerular hili, arterioles, and arterial branches was significantly higher in the cases with diabetes than in the control cases without diabetes $(P \leq 0.001)$. Furthermore, among the cases with diabetes, the cases with DN had a significantly higher prevalence of $\mathrm{Clq}$ in the glomerular hili and arterioles compared with the cases without DN $(P<0.05)$, whereas the prevalence of $\mathrm{Clq}$ in the arterial branches did not differ significantly between these 2 groups ( $p=0.106)$. Finally, the presence of $\mathrm{Clq}$ deposits was correlated with the presence of $\mathrm{C} 4 \mathrm{~d}$ deposits in the glomeruli $(P=0.006)$, glomerular hili $(P=0.027)$, and arterioles $(P<0.001)$.

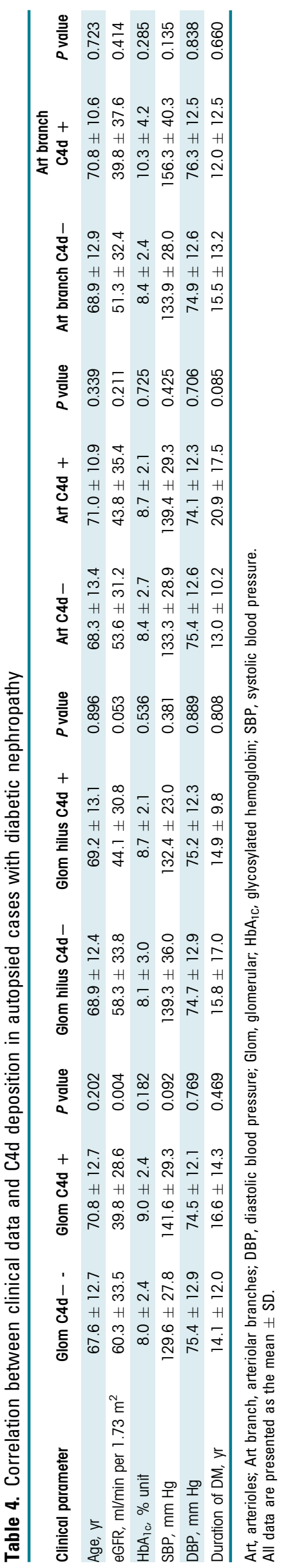

Kidney International Reports (2018) 3, 302-313 


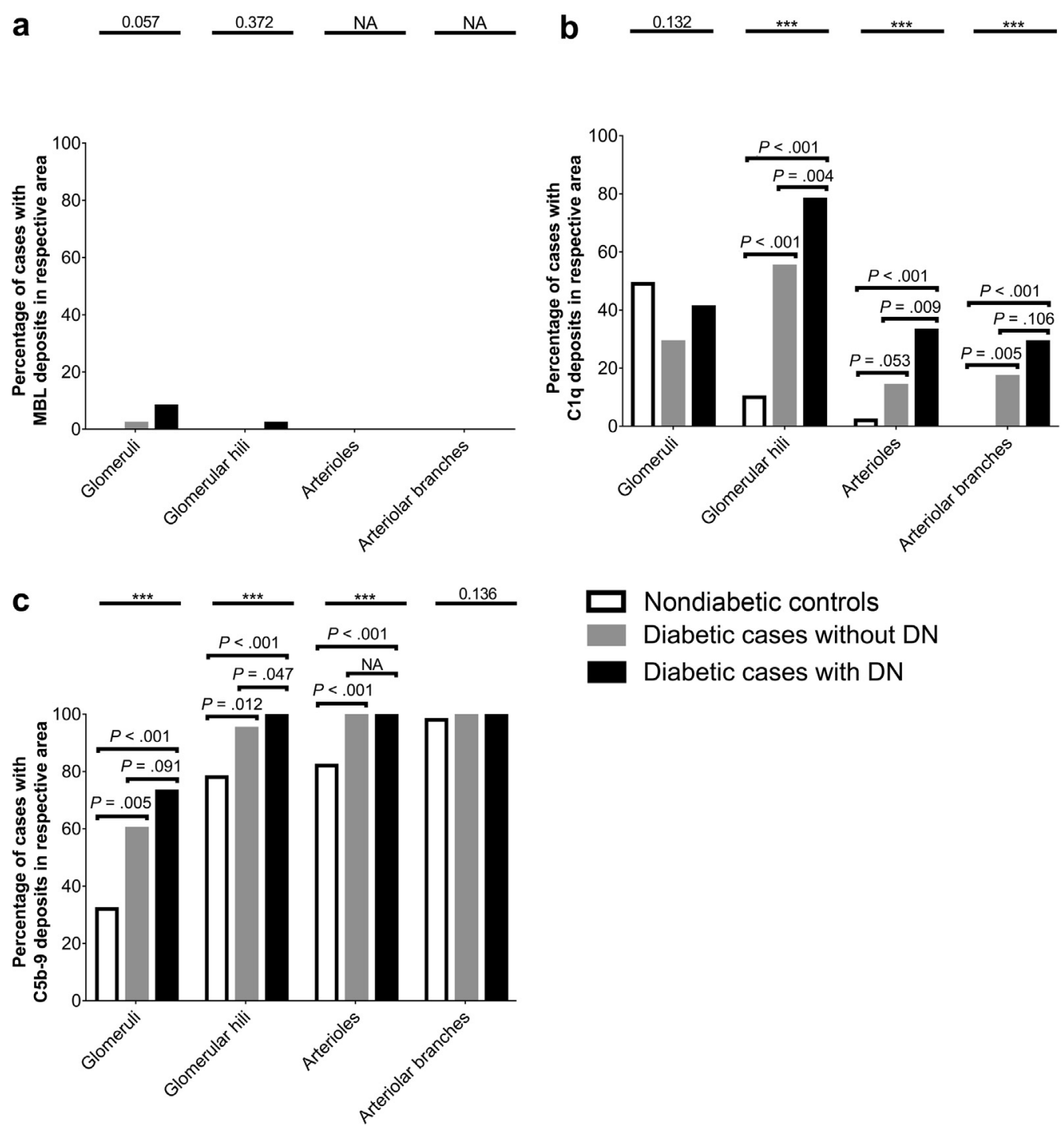

Figure 2. $(a-c)$ Prevalence of mannose-binding lection (MBL), C1q, and C5b-9 deposits in cases and controls. The percentage of cases and controls with complement factor MBL (a), C1q (b), and/or C5b-9 (c) is shown for the indicated renal structures. The $P$ values shown between the 2 groups represent post hoc analyses. ${ }^{* *} P<0.001, \chi^{2}$ test between nondiabetic controls, diabetic cases without diabetes nephropathy (DN), and diabetic cases with DN.

\section{C5b-9 Deposits Are Associated With Diabetes, But Not With DN}

In the cases with diabetes, glomerular C5b-9 staining was predominantly diffuse and global. Although the prevalence of C5b-9 deposits was significantly higher in the glomeruli, glomerular hili, and arterioles of the cases with diabetes compared with the control cases without diabetes $(P<0.001)$ (Figure $2 \mathrm{c}$ and Table 3$)$, the prevalence of C5b-9 was still relatively high in the control cases without diabetes. Only the prevalence of C5b-9 deposits in the glomerular hili were significantly higher in the cases with DN than in cases without DN $(P=0.047)$ (Figure $2 \mathrm{c})$. The presence of glomerular C5b-9 deposits was correlated with the presence of glomerular C4d deposits $(P=0.002)$.

The prevalence, location, distribution, and staining patterns of complement proteins in the glomeruli, glomerular hili, arterioles, and arterial branches of the cases with diabetes are listed in Table 3. Representative images of Clq, C4d, MBL, and C5b-9 staining in these structures are shown in Figure 3. C4d and C5b-9 deposits are correlated with histological lesions and DN class.

Among the cases with diabetes, the presence of glomerular $\mathrm{C} 4 \mathrm{~d}$ was correlated with glomerular hyalinosis $(P=0.020)$, IFTA $(P<0.001)$, arteriosclerosis $(P=0.017)$, and arteriolar hyalinosis $(P=0.017)$ (Supplemental Table S1A and S1B). Moreover, the presence of $\mathrm{C} 4 \mathrm{~d}$ in the glomerular hili was correlated with glomerular hyalinosis $(P=0.002)$, and the presence of C4d in the arterioles was correlated with IFTA $(P<0.001)$, arteriosclerosis $(P=0.041)$, and arteriolar hyalinosis $(P=0.041)$. Although glomerular $\mathrm{Clq}$ was not correlated with histological lesions, the presence of 

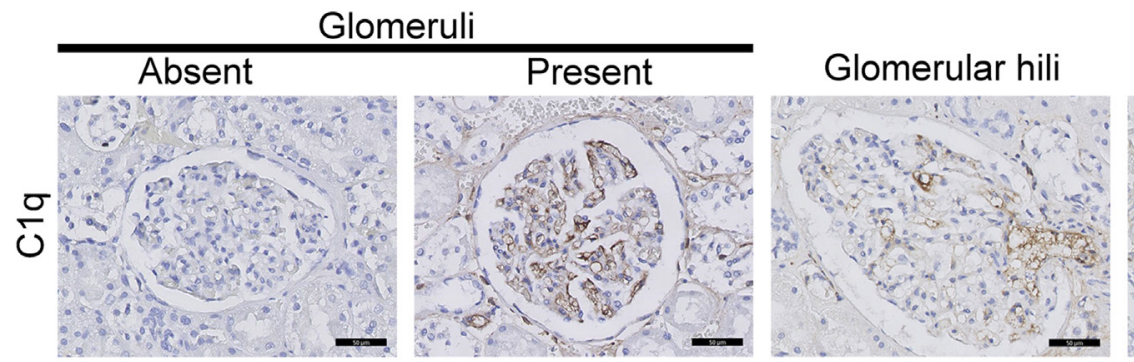

Arterioles $/$
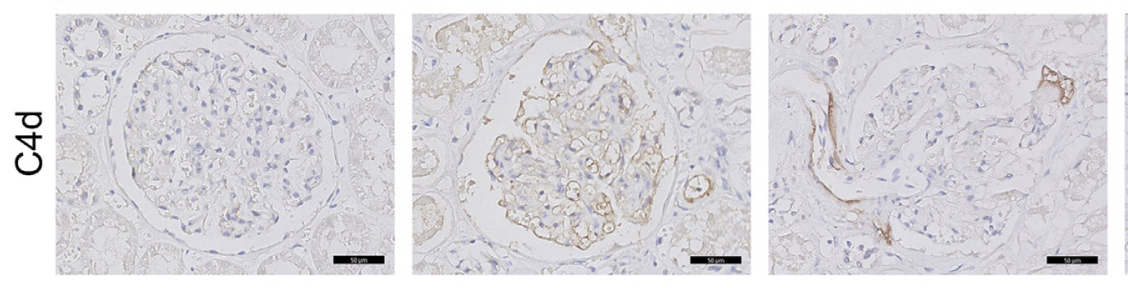

Arterial branches
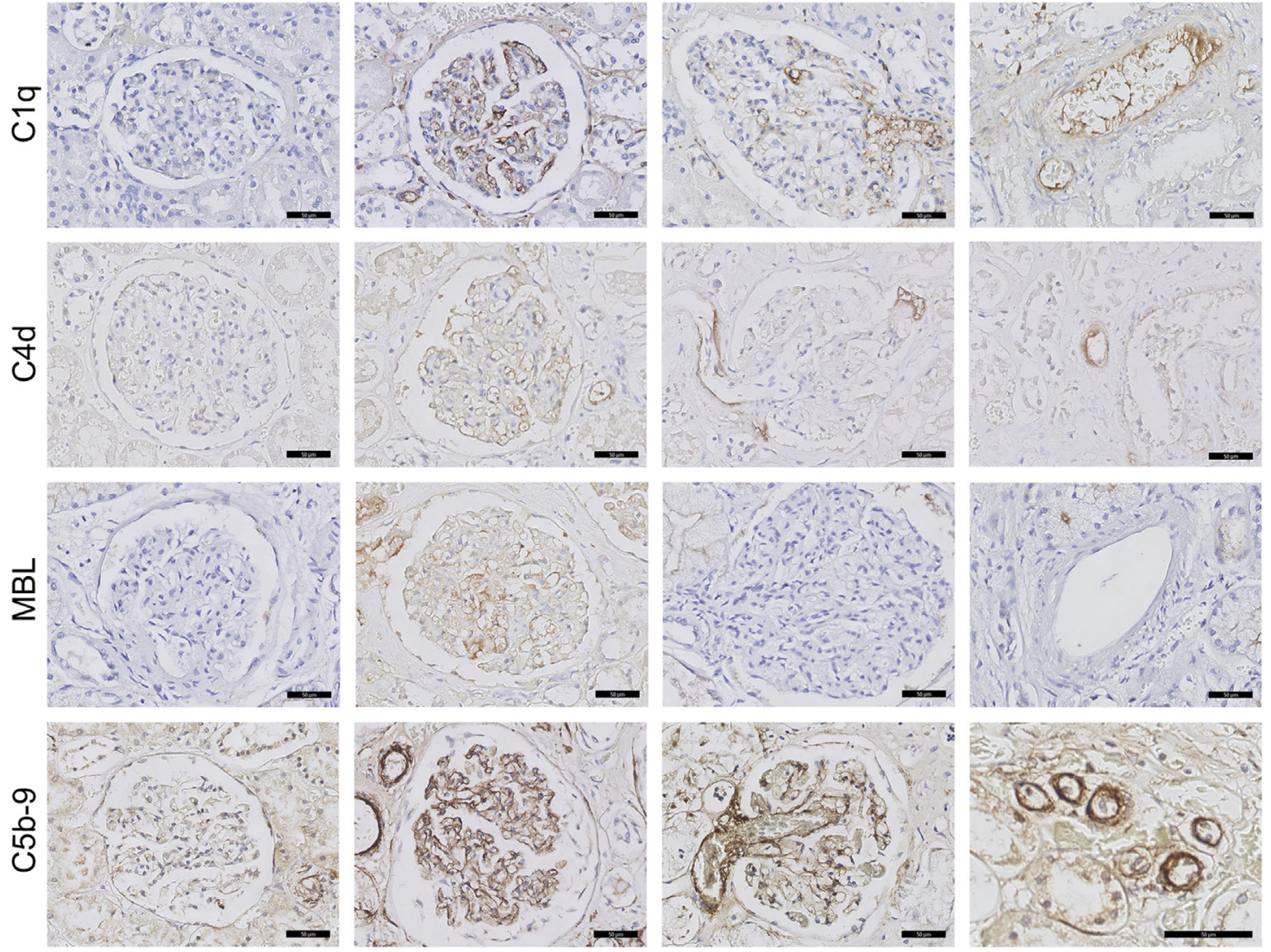

Figure 3. Representative images of complement staining in patients. Kidney sections were immunostained for the indicated proteins, and representative images containing the glomeruli, glomerular hili, arterioles, and arterial branches are shown. Mannose-binding lection $(\mathrm{MBL})$ staining was negative in the glomerular hilus, arterioles, and arterial branches. Bars $=50 \mu \mathrm{m}$.

$\mathrm{Clq}$ in the arterioles was correlated with the presence of glomerular capsular drop $(P=0.034)$, arteriolar hyalinosis $(P=0.048)$, and IFTA $(P=0.027)$. The presence of MBL in the glomeruli, arterioles, and arterial branches was not correlated with any renal lesion. In addition, the presence of glomerular C5b-9 was correlated with IFTA $(P=0.008)$. Finally, with respect to DN class, the prevalence of glomerular C4d and C5b-9 was correlated with more severe classes of
DN ( $p \leq 0.001$ ) (Figure 4), particularly with class III and class IV DN.

\section{Differences in Complement Deposition Between Type 1 DM and Type 2 DM}

Data on diabetes type was available for 137 cases $(86 \%$ of cases with diabetes); 17 cases (12\%) had type $1 \mathrm{DM}$ and 120 cases $(88 \%)$ had type 2 DM. Compared with cases with type $2 \mathrm{DM}$, cases with type $1 \mathrm{DM}$ had a
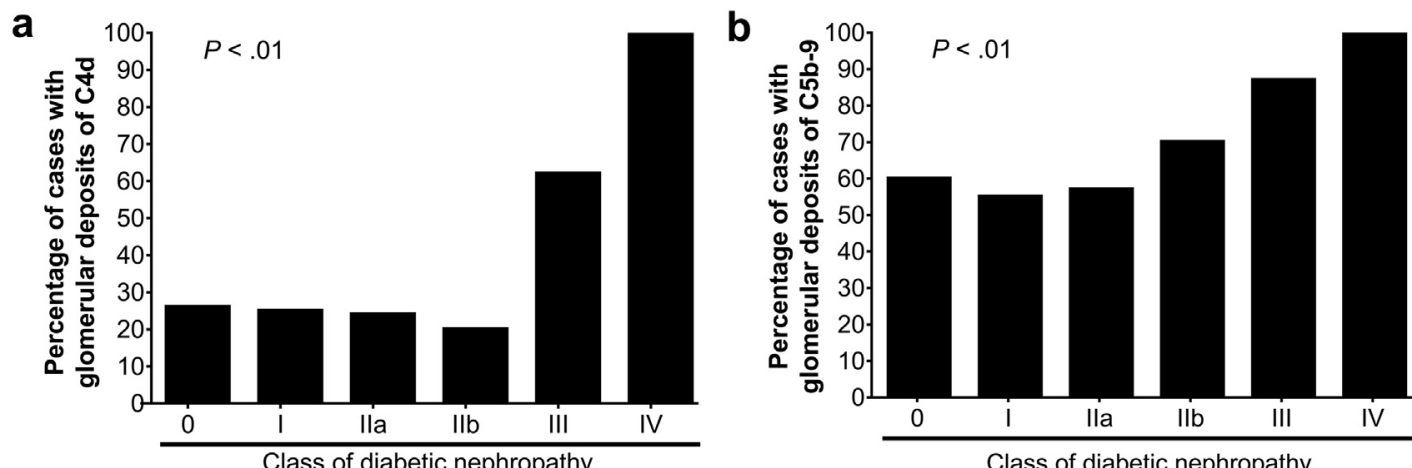

Figure 4. Percentage of patients with glomerular C4d deposits and glomerular C5b-9 deposits plotted against diabetic nephropathy class. The presence of glomerular C4d (a) and C5b-9 (b) was correlated with diabetic nephropathy class. For C4d and C5b-9, the Spearman rank correlation coefficient $(\rho)$ was 0.344 and 0.228 , respectively (both $P<0.01$ ). Cases without diabetic nephropathy were classified as class 0 . 
significantly higher prevalence of $\mathrm{C} 4 \mathrm{~d}$ in glomeruli $(64 \%$ vs. $33 \% ; P=0.012)$, C4d in arterioles $(47 \%$ vs. $18 \% ; P=0.007)$, and C5b-9 in glomeruli $(94 \%$ vs. $64 \% ; P=0.013)$. Within the subgroup of cases with DN, cases with type 1 DM also had a significantly higher prevalence of $\mathrm{C} 4 \mathrm{~d}$ in glomeruli $(75 \%$ vs. $35 \%$; $P<0.01)$, C4d in arterioles (50\% vs. $22 \% ; P=0.040)$, and C5b-9 in glomeruli $(100 \%$ vs. $68 \% ; P<0.05)$.

\section{Evidence for the Activation of the Classical Complement Pathway in Biopsies}

To support our findings with autopsy tissue, we found that the prevalence of glomerular C4d was significantly higher in biopsies from cases with DN compared with a control group of biopsies obtained from healthy living transplantation donors $(75 \%$ vs. $10 \%$, respectively; $P=0.002)$; similar results were obtained with respect to glomerular C5b-9 deposits $(75 \%$ vs. $0 \%$, respectively; $P=0.001$ ) (Figure 5). In contrast, the prevalence of glomerular $\mathrm{Clq}$ deposits did not differ significantly between these 2 groups (58\% vs. $40 \%$, respectively; $P=0.392$ ), and glomerular MBL deposits were not observed in either group. The prevalence of glomerular IgM deposits was significantly higher in the cases with DN compared with the control cases $(82 \%$ vs. $30 \%$, respectively; $P=0.017)$, and the prevalence of glomerular IgM deposits were significantly correlated with the prevalence of glomerular Clq $(P=0.003)$ and $\mathrm{C} 4 \mathrm{~d}(P=0.001)$ deposits. Finally, glomerular IgM deposits co-localized with glomerular $\mathrm{Clq}$ and C4d deposits (Figure 6), which suggested that the classical complement pathway was activated in cases with DN.

\section{DISCUSSION}

$\mathrm{DN}$ is a microvascular complication that affects $20 \%$ to $40 \%$ of patients with diabetes, making it the leading cause of end-stage renal disease. ${ }^{1-3}$ An increasing body

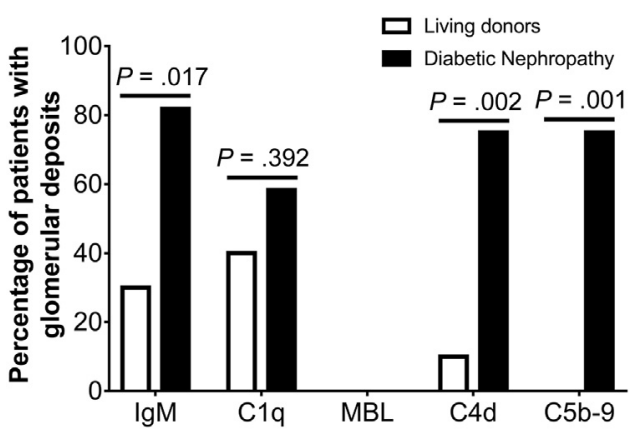

Figure 5. Percentage of renal sections containing $\lg M, C 1 q$, mannose-binding lectin (MBL), C4d, or C5b-9 deposits. Biopsy samples were obtained from living patients with diabetic nephropathy $(\mathrm{n}=12$ ) and healthy living renal transplantation donors ( $\mathrm{n}=10$ ) and stained for $\operatorname{lgM}, \mathrm{Clq}, \mathrm{MBL}, \mathrm{C} 4 \mathrm{~d}$, and $\mathrm{C} 5 \mathrm{~b}-9 . P$ values were calculated using the $\chi^{2}$ test. of evidence has suggested that complement activation via the lectin and/or classical complement pathway plays a role in the development of $\mathrm{DN} \cdot{ }^{4-7,14} \mathrm{We}$ investigated the deposition of complement proteins in a relatively large cohort of cases with diabetes with DN and without DN. We found that the complement activation marker C4d was correlated with $\mathrm{DN}$, as well as with the severity of DN, microvascular and interstitial lesions, and lower eGFR in cases with DN, suggesting that complement activation might play a role in the development of DN.

Because C4d binds covalently to its target cells, C4d can be observed long after the factors that activated the pathway have dissociated, making it a commonly used biomarker for complement activation. ${ }^{22}$ The prevalence of C4d deposits in the glomeruli and arterioles was significantly higher in cases with DN than cases without DN and in cases without diabetes without renal pathology, both in the autopsy cohort and in the biopsy cohort. Moreover, glomerular C4d and arteriolar C4d were more prevalent in the cases with vascular and chronic renal lesions, and glomerular $\mathrm{C} 4 \mathrm{~d}$ was correlated with the severity of DN. These data suggested that complement activation, together with the renal microvasculature, might be involved in the development of DN. This notion was supported by the general absence of C4d deposits among the control cases without diabetes.

To investigate which complement pathway could underlie the deposition of $\mathrm{C} 4 \mathrm{~d}$, we studied the prevalence and localization of $\mathrm{Clq}$ (to measure the classical pathway) and MBL (to measure the lectin pathway) deposits. The presence of $\mathrm{Clq}$, IgM, and C4d deposits were associated with each other, and co-localized in the same renal vascular structures, whereas MBL was detected rarely in our cohort. These findings suggested that the presence of $\mathrm{C} 4 \mathrm{~d}$ reflected activation of the classical complement pathway. However, because MBL is not covalently bound and because other proteins such as ficolins can initiate the lectin pathway, we could not exclude the possibility that activation of the lectin pathway led to C4d deposition.

C4d deposits were significantly more prevalent in kidneys from cases with type $1 \mathrm{DM}$ than in kidneys from cases with type $2 \mathrm{DM}$, both in the total cohort of cases with diabetes and in the subgroup of cases with DN. The presence of DN was not a confounder in this association because DN was not more prevalent in type 1 DM compared with type 2 DM. Rowe et al. showed similar differences in pancreatic C4d deposition among cases with type $1 \mathrm{DM}$, type $2 \mathrm{DM}$, and control cases without diabetes. ${ }^{23}$ The higher $\mathrm{C} 4 \mathrm{~d}$ prevalence between cases with type $1 \mathrm{DM}$ and type 1 DM could possibly reflect a different pathogenesis. Nevertheless, 

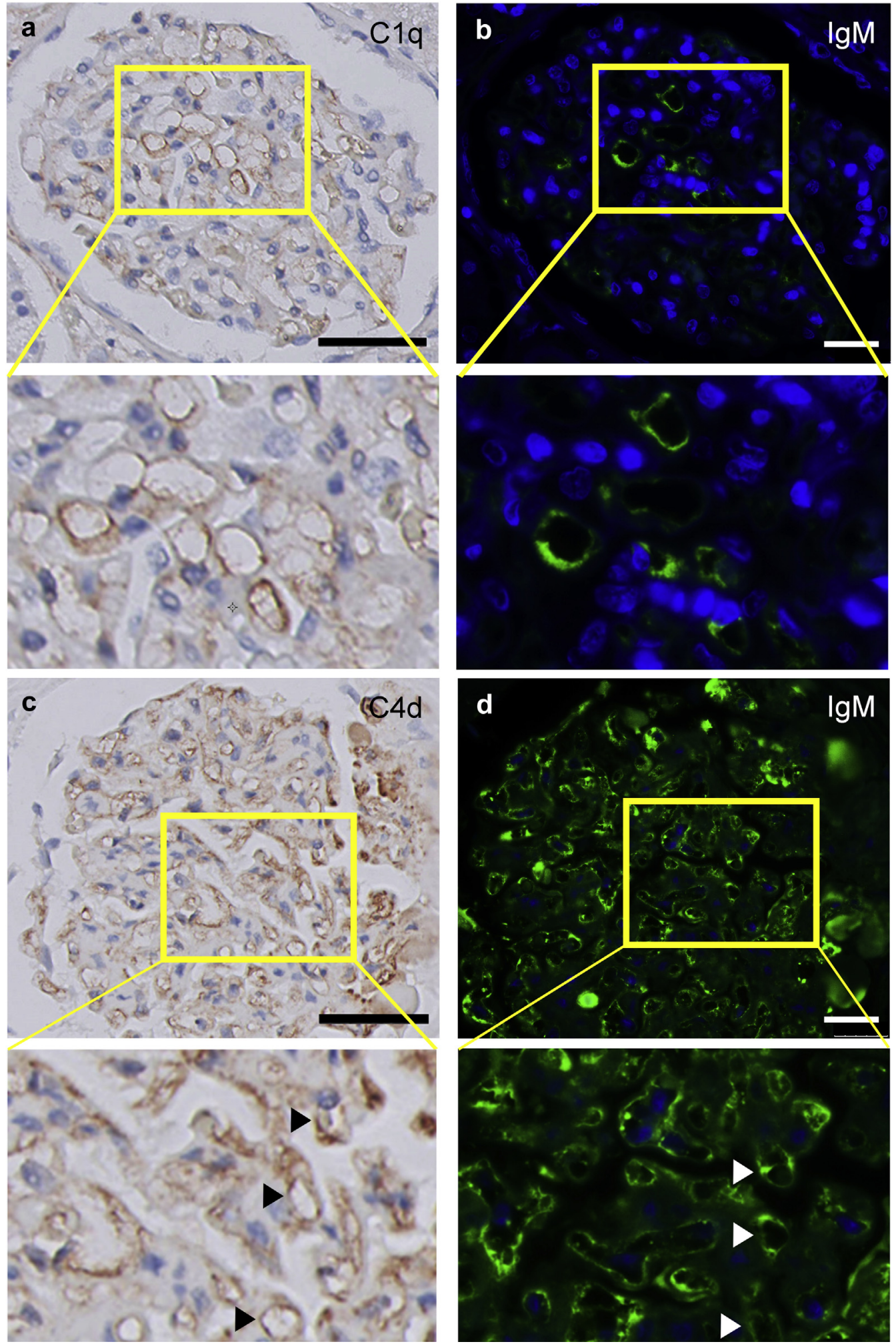

Figure 6. Glomerular IgM deposits co-localize with glomerular C1q and C4d. Adjacent sections of an autopsied kidney from a case with diabetic nephropathy were stained for $\mathrm{C1q}(\mathrm{a})$ or $\operatorname{IgM}(\mathrm{b})$. Adjacent sections of an autopsied kidney from a case with diabetic nephropathy were stained for C4d (c) or IgM (d); the arrowheads indicate co-localization between C4d and IgM. The scale bars in (a) and (c) represent $50 \mu \mathrm{m}$; the scale bars in (b) and (d) represent $25 \mu \mathrm{m}$.

we could not exclude the possibility that the difference observed in complement deposition might be attributed, at least in part, to the duration of diabetes rather than the type of diabetes.
Glomerular C4d was a common finding in our cases with class III DN. Paueksakon et al. suggested that Kimmelstiel-Wilson lesions, which are a hallmark lesion of DN and a diagnostic requirement for class III DN, 
might be a form of thrombotic microangiopathy. Specifically, they found that a subset of cases with DN had fragmented red blood cells exclusively in KimmelstielWilson lesions. ${ }^{24}$ Recently, we reported that complement factor C4d was a common denominator in several thrombotic microangiopathies. ${ }^{25}$ Thus, our data further supported the hypothesis that Kimmelstiel-Wilson lesions might be a form of thrombotic microangiopathy that arises from complement activation. The underlying cause of C4d deposits in the arterioles, arteries, and glomerular hilus (the junction between afferent and efferent arterioles) is currently unknown. Because both renal afferent arterioles and renal efferent arterioles play a role in regulating renal blood flow, these vessels can be exposed to extremely turbulent blood flow and high shear stress conditions, ${ }^{26-29}$ which may increase vulnerability to vascular injury, complement deposition, and/or the development of renal pathology.

We found that C5b-9 deposits were more prevalent in our cases with diabetes than in the control cases without diabetes; however, the clinical relevance of these deposits in our cohort was difficult to interpret, because C5b-9 was relatively frequently prevalent in control cases without diabetes (albeit to a lesser extent than in the cases with diabetes). This finding might be related in part to our use of autopsy samples because we did not observe these deposits in renal biopsies obtained from living donors, which was consistent with findings reported by Qin et al. ${ }^{11}$ Nevertheless, in our cohort of cases with diabetes with $\mathrm{DN}$, the presence of glomerular C5b-9 was correlated with the severity of DN, which suggested that C5b-9 might play a role in the progression of renal damage. Our data suggested that the process that leads to $\mathrm{C} 4 \mathrm{~d}$ deposition also leads to C5b-9 deposition. However, C5b-9 could have also been activated due to a direct effect of hyperglycemia on regulatory proteins in the complement system. Hyperglycemia can lead to the glycation of CD59, which inhibits C5b-9 under physiological conditions. ${ }^{11,18}$ This glycation-induced inactivation of CD59 could lead to the formation of C5b-9.

In the setting of DN, several factors could have led to the deposition of C4d. For example, autoantibodies can activate the complement system. ${ }^{30}$ This notion is supported by the report of linear IgG staining along the glomerular basement membrane in $60 \%$ of type $2 \mathrm{DM}$ cases with DN. ${ }^{31}$ In addition, high glucose levels in cases with DM can lead to increased levels of glycated proteins, including advanced glycation end-products and oxidized proteins, ${ }^{32,33}$ which can activate the classical and/or lectin pathways either directly or by reacting with autoantibodies. ${ }^{16,34-36}$ The complement system can also be activated by the binding of natural (i.e., $\operatorname{Ig} M$ ) antibodies to either hypoxic or apoptotic cells in the setting of DN. ${ }^{37-39}$ Natural antibodies play an important role in clearing damaged cells via intracellular antigens that are externalized during apoptosis and/or hypoxic conditions. ${ }^{37-41}$ We found a significantly higher prevalence of glomerular IgM deposits in biopsies from cases with DN compared with healthy living transplantation donors. Moreover, glomerular IgM deposits co-localized with - and were significantly correlated with-both $\mathrm{Clq}$ and $\mathrm{C} 4 \mathrm{~d}$, which supported the hypothesis that IgM antibodies activate the classical complement pathway in cases with DN.

It is currently unknown whether complement activation is a cause and/or consequence of microvascular damage in DN. Our data suggested that complement activation was involved in the progression of renal damage in cases with diabetes mellitus because complement activation was correlated with more severe renal damage, including higher DN class and increased IFTA levels. These findings were consistent with other studies with regard to complement activation in the development of $\mathrm{DN}$ and other diabetes-associated microvascular and/or macrovascular complications. ${ }^{7,14}$ Furthermore, type 2 diabetic rats treated with a complement inhibitor had improved renal function and morphology compared with untreated rats, ${ }^{19}$ which supported the notion that complement activation plays a role in the progression of diabetes-associated kidney disease. In the context of DM, hyperglycemia can both directly and indirectly lead to complement deposition, which can then lead to the increased production of reactive oxygen species, activation of protein kinase $C$, and upregulation of nuclear factor- $\kappa \mathrm{B}$, thereby inducing the release of proinflammatory, prothrombotic cytokines, and growth factors. ${ }^{7}$ Both complement-dependent and complement-independent mechanisms can then lead to inflammation, proliferation, and thrombosis, which together characterize the diabetes-associated complications in target organs. In contrast, complement activation may also be a consequence of renal vascular damage, possibly following activation via natural antibodies. This hypothesis is consistent with our findings regarding the presence of C4d and IgM antibodies in other renal microangiopathies. $^{25,42}$ Moreover, we previously reported that C4d deposits were associated with remodeling of the glomerular basement membrane, ${ }^{43}$ which was consistent with cases with DN that presented with a remodeled glomerular basement membrane. Nevertheless, despite our relatively large cohort and our ability to examine $>100$ glomeruli per case, the autopsy-based nature of this study precluded our ability to measure causality. Therefore, future studies should be designed to determine whether complement activation is a cause, consequence, or mediator of DN. 
Our study had several limitations. First, the use of autopsy samples precluded our ability to investigate whether the prevalence of complement deposition in the glomeruli, glomerular hili, and/or vessels was associated with patient survival and/or renal survival. Second, our study might have had a selection bias because not all cases with diabetes had an autopsy; generally speaking, most cases that are autopsied were in the hospital at the time of death. Third, because autopsy samples were used, we could not exclude the possibility that postmortem changes and/or cause of death might have affected the tissue in terms of protein expression and/or tissue morphology. However, to address this possibility, we examined control samples obtained from autopsied cases without diabetes or renal pathology. Furthermore, because cause of death was not significantly correlated with the prevalence of complement deposits, and because our findings were supported by examining renal biopsy samples, we concluded that our data were not likely affected by autopsy-related artifacts. In contrast, the strength of our study was the relatively high number of cases combined with our ability to examine $>100$ glomeruli per case.

In conclusion, complement activation is associated with DN, and both glomerular C4d and glomerular C5b-9 deposits are correlated with the class of DN. Furthermore, complement deposits in several renal vascular structures are correlated with more severe renal damage. Our data suggest that complement activation is involved with the development of renal damage in cases with diabetes, and that inhibition or modulation of complement activity could be a promising therapeutic strategy for patients with DN.

\section{DISCLOSURE}

All the authors declared no competing interests.

\section{ACKNOWLEDGMENTS}

We would like to thank Dr. Ingeborg Bajema for critically evaluating this manuscript.

\section{SUPPLEMENTARY MATERIAL}

Table S1. (A) C1q deposits and histologic lesions in all diabetic cases. (B) mannose-binding lection (MBL) deposits and histologic lesions in all diabetic cases. (C) C4d deposits and histologic lesions in all diabetic cases. (D) C5b-9 deposits and histologic lesions in all diabetic cases. Supplementary material is linked to the online version of the paper at www.kireports.org.

\section{REFERENCES}

1. Andersen AR, Christiansen JS, Andersen JK, et al. Diabetic nephropathy in type 1 (insulin-dependent) diabetes: an epidemiological study. Diabetologia. 1983;25:496-501.
2. Adler Al, Stevens RJ, Manley SE, et al. Development and progression of nephropathy in type 2 diabetes: the United Kingdom Prospective Diabetes Study (UKPDS 64). Kidney Int. 2003;63:225-232.

3. Tryggvason G, Indridason OS, Thorsson AV, et al. Unchanged incidence of diabetic nephropathy in type 1 diabetes: a nation-wide study in Iceland. Diabet Med. 2005;22:182-187.

4. Valencia WM, Florez H. How to prevent the microvascular complications of type 2 diabetes beyond glucose control. BMJ. 2017;356:i6505.

5. Atkinson MA, Eisenbarth GS, Michels AW. Type 1 diabetes. Lancet. 2014;383:69-82.

6. Schrijvers BF, De Vriese AS, Flyvbjerg A. From hyperglycemia to diabetic kidney disease: the role of metabolic, hemodynamic, intracellular factors and growth factors/cytokines. Endocr Rev. 2004;25:971-1010.

7. Ghosh P, Sahoo R, Vaidya A, et al. Role of complement and complement regulatory proteins in the complications of diabetes. Endocr Rev. 2015;36:272-288.

8. Woroniecka KI, Park AS, Mohtat D, et al. Transcriptome analysis of human diabetic kidney disease. Diabetes. 2011;60:2354-2369.

9. Ricardo SD, van Goor H, Eddy AA. Macrophage diversity in renal injury and repair. J Clin Invest. 2008;118:3522-3530.

10. Falk RJ, Dalmasso AP, Kim Y, et al. Neoantigen of the polymerized ninth component of complement. Characterization of a monoclonal antibody and immunohistochemical localization in renal disease. J Clin Invest. 1983;72:560-573.

11. Qin X, Goldfine A, Krumrei N, et al. Glycation inactivation of the complement regulatory protein CD59: a possible role in the pathogenesis of the vascular complications of human diabetes. Diabetes. 2004;53:2653-2661.

12. Ogrodowski JL, Hebert LA, Sedmak D, et al. Measurement of SC5b-9 in urine in patients with the nephrotic syndrome. Kidney Int. 1991;40:1141-1147.

13. Morita $\mathrm{Y}$, Ikeguchi H, Nakamura J, et al. Complement activation products in the urine from proteinuric patients. J Am Soc Nephrol. 2000;11:700-707.

14. Flyvbjerg A. The role of the complement system in diabetic nephropathy. Nat Rev Nephrol. 2017;13:311-318.

15. Saraheimo M, Forsblom C, Hansen TK, et al. Increased levels of mannan-binding lectin in type 1 diabetic patients with incipient and overt nephropathy. Diabetologia. 2005;48:198-202.

16. Chikazawa $\mathrm{M}$, Shibata $\mathrm{T}$, Hatasa $\mathrm{Y}$, et al. Identification of $\mathrm{C} 1 \mathrm{q}$ as a binding protein for advanced glycation end products. Biochemistry. 2016;55:435-446.

17. Kelly KJ, Liu Y, Zhang J, et al. Renal C3 complement component: feed forward to diabetic kidney disease. Am J Nephrol. 2015;41:48-56.

18. Ghosh P, Sahoo R, Vaidya A, et al. A specific and sensitive assay for blood levels of glycated CD59: a novel biomarker for diabetes. Am J Hematol. 2013;88:670-676.

19. Li L, Yin Q, Tang X, et al. C3a receptor antagonist ameliorates inflammatory and fibrotic signals in type 2 diabetic nephropathy by suppressing the activation of TGF-beta/ smad3 and IKBalpha pathway. PLoS One. 2014;9:e113639.

20. Klessens CQ, Woutman TD, Veraar KA, et al. An autopsy study suggests that diabetic nephropathy is underdiagnosed. Kidney Int. 2016;90:149-156. 
21. Tervaert TW, Mooyaart AL, Amann K, et al. Pathologic classification of diabetic nephropathy. J Am Soc Nephrol. 2010;21:556-563.

22. Cohen D, Colvin RB, Daha MR, et al. Pros and cons for C4d as a biomarker. Kidney Int. 2012;81:628-639.

23. Rowe P, Wasserfall C, Croker B, et al. Increased complement activation in human type 1 diabetes pancreata. Diabetes Care. 2013;36:3815-3817.

24. Paueksakon P, Revelo MP, Ma LJ, et al. Microangiopathic injury and augmented PAI-1 in human diabetic nephropathy. Kidney Int. 2002;61:2142-2148.

25. Chua JS, Baelde HJ, Zandbergen M, et al. Complement factor $\mathrm{C} 4 \mathrm{~d}$ is a common denominator in thrombotic microangiopathy. J Am Soc Nephrol. 2015;26:2239-2247.

26. Lipowsky HH, Kovalcheck S, Zweifach BW. The distribution of blood rheological parameters in the microvasculature of cat mesentery. Circ Res. 1978;43:738-749.

27. Papaioannou TG, Stefanadis C. Vascular wall shear stress: basic principles and methods. Hellenic J Cardiol. 2005;46:9-15.

28. Loutzenhiser R, Griffin KA, Bidani AK. Systolic blood pressure as the trigger for the renal myogenic response: protective or autoregulatory? Curr Opin Nephrol Hypertens. 2006;15:41-49.

29. Endlich K, Muller C, Barthelmebs M, et al. Role of shear stress in nitric oxide-dependent modulation of renal angiotensin II vasoconstriction. Br J Pharmacol. 1999;127:1929-1935.

30. Dalvin LA, Fervenza FC, Sethi S, et al. Manifestations of complement-mediated and immune complex-mediated membranoproliferative glomerulonephritis: a comparative consecutive series. Ophthalmology. 2016;123:1588-1594.

31. Mazzucco G, Bertani T, Fortunato M, et al. Different patterns of renal damage in type 2 diabetes mellitus: a multicentric study on 393 biopsies. Am J Kidney Dis. 2002;39:713-720.

32. Orchard TJ, Virella G, Forrest KY, et al. Antibodies to oxidized LDL predict coronary artery disease in type 1 diabetes: a nested case-control study from the Pittsburgh Epidemiology of Diabetes Complications Study. Diabetes. 1999;48:1454-1458.
33. Giacco F, Brownlee M. Oxidative stress and diabetic complications. Circ Res. 2010;107:1058-1070.

34. Witztum JL, Steinbrecher UP, Fisher M, et al. Nonenzymatic glucosylation of homologous low density lipoprotein and albumin renders them immunogenic in the guinea pig. Proc Natl Acad Sci U S A. 1983;80:2757-2761.

35. Malhotra A, Lopez MC, Nakouzi A. Troponin subunits contribute to altered myosin ATPase activity in diabetic cardiomyopathy. Mol Cell Biochem. 1995;151:165-172.

36. Fortpied J, Vertommen D, Van Schaftingen E. Binding of mannose-binding lectin to fructosamines: a potential link between hyperglycaemia and complement activation in diabetes. Diabetes Metab Res Rev. 2010;26:254-260.

37. Strassheim D, Renner B, Panzer S, et al. IgM contributes to glomerular injury in FSGS. J Am Soc Nephrol. 2013;24:393-406.

38. van der Pol P, Roos A, Berger SP, et al. Natural IgM antibodies are involved in the activation of complement by hypoxic human tubular cells. Am J Physiol Renal Physiol. 2011;300:F932-F940.

39. Peng $Y$, Kowalewski $\mathrm{R}$, Kim $\mathrm{S}$, et al. The role of IgM antibodies in the recognition and clearance of apoptotic cells. Mol Immunol. 2005;42:781-787.

40. Fu M, Fan PS, Li W, et al. Identification of poly-reactive natural IgM antibody that recognizes late apoptotic cells and promotes phagocytosis of the cells. Apoptosis. 2007;12:355-362.

41. Vollmers HP, Brandlein S. Natural human immunoglobulins in cancer immunotherapy. Immunotherapy. 2009;1: 241-248.

42. Penning M, Chua JS, van Kooten C, et al. Classical complement pathway activation in the kidneys of women with preeclampsia. Hypertension. 2015;66:117-125.

43. Gasim AH, Chua JS, Wolterbeek R, et al. Glomerular C4d deposits can mark structural capillary wall remodelling in thrombotic microangiopathy and transplant glomerulopathy: C4d beyond active antibody-mediated injury: a retrospective study. Transpl Int. 2017;30:519-532. 